\title{
A fast scatter field estimator for Digital Breast Tomosynthesis
}

\author{
Oliver Díaz ${ }^{a}$, David R. Dance ${ }^{b, c}$, Kenneth C. Young ${ }^{b, c}$, Premkumar Elangovan ${ }^{a}$, Predrag R. \\ Bakic $^{d}$ and Kevin Wells ${ }^{a}$ \\ ${ }^{a}$ Centre for Vision, Speech and Signal Processing, University of Surrey, Guildford, GU2 7XH, \\ $\mathrm{UK}$; \\ ${ }^{b}$ National Coordinating Centre for the Physics of Mammography, Royal Surrey County \\ Hospital, Guildford, GU2 7XX, UK; \\ ${ }^{c}$ Dept. of Physics, Faculty of Engineering and Physical Sciences, University of Surrey, \\ Guildford, GU2 7XH, UK; \\ ${ }^{d}$ Dept. of Radiology, University of Pennsylvania, Philadelphia, PA 19104, USA
}

\begin{abstract}
Digital breast tomosynthesis (DBT) is a promising alternative approach to overcome the limitations of tissue superposition found in full-field 2D digital mammography. However, due to the absence of anti-scatter grids in DBT, accurate scatter estimation for each projection is necessary for modelling the image reconstruction stage. In this work we identify the limitations associated with scatter estimation using spatial invariant scatter kernels, in particular at the edge region where such methods result in scatter overestimation. Such approaches show an overestimation of scatter-to-primary ratio of over $50 \%$ at the edges when compared with results from direct Monte Carlo simulation. This problem was found to increase with projection angle. Simulation work presented here shows that this overestimation in scatter is largely due to air gap between the lower curved breast edge and the detector.

We propose a new fast, accurate scatter field estimator for use in DBT which not only considers the breast thickness and primary incidence angle, but also accounts for scatter exiting the breast edge region and traversing an air gap prior to absorption in the detector. The new proposed scatter estimator represents an alternative approach to this problem which reduces discrepancies at the edge of a breast phantom. Moreover, the time required for generating scatter has dropped from approximately 12 hours using Monte Carlo simulations for $10^{10}$ photons to just a few minutes per projection. The insertion of scatter from the compression paddle to aforementioned methodologies is also discussed.
\end{abstract}

Keywords: Scatter correction, digital breast tomosynthesis, Monte Carlo simulations, convolution method Presented at SPIE'2012 (San Diego, USA)

doi: $10.1117 / 12.911494$

url: http://proceedings.spiedigitallibrary.org/proceeding.aspx?articleid=1386326

\section{INTRODUCTION}

X-ray mammography is the standard imaging technique for population screening for breast cancer. However, because it produces a 2D projected image of the breast, it suffers from limitations due to the superposition in the image of breast anatomical structures, which may obfuscate and/or simulate the appearance of a lesion. Digital breast tomosynthesis (DBT) has the potential to overcome this limitation. It uses a series of 2D projections of the breast taken at different (but limited) angles which are reconstructed in a series of parallel planes, thus allowing the visualisation of depth information within the breast. One of the problems in using DBT is the absence of an anti-scatter grid which leads to a significantly increased amount of scatter recorded in each projection image. Thus scatter fields need to be estimated as part of any correction scheme to reduce the contrast-reducing effect of this undesirable component and to minimise the associated error during reconstruction $^{1}$. Knowledge of this field is also required for performance studies using simulation.

\footnotetext{
Further author information: (Send correspondence to Oliver Díaz)

Oliver Díaz: E-mail: o.diaz@surrey.ac.uk
} 
Scatter can be estimated by Monte Carlo (MC) simulations, but this is extremely time-consuming, and it is therefore attractive to use a faster convolution-based method of scatter estimation ${ }^{1-3}$, with an appropriate scatter kernel. We have tested this approach by comparing direct MC calculations of the scatter-to-primary ratio (SPR) obtained when using a realistic anthropomorphic phantom to simulate the breast and convolution-based calculations with scatter kernels determined for breast phantoms of uniform thickness and composition. A novel approach is suggested which shows significantly improved accuracy when the air gap between the lower curved breast boundary and the image receptor is considered when defining an appropriate scatter kernel. This occurs towards the edge of the breast, and has an important influence on the amount of scatter recorded. Scattered radiation from the compression paddle is also important, though discussed in the literature. A preliminary methodology for allowing for this is also shown.

\section{MATERIALS AND METHODOLOGY}

Monte Carlo simulations have been undertaken using Geant $4^{4,5}$. Simulations were made for the direct calculation of the SPR for full-field irradiation of an anthropomorphic phantom and for the calculation of scatter response functions (which are used as kernels for the convolution algorithm) using pencil beam irradiation of uniform phantoms. The simulations have also been used to estimate a correction factor for use with the convolution algorithm and to study the scatter from the compression paddle. The statistical error measured in the MC simulations when using the anthropomorphic phantom was approximately $4 \%$.

\subsection{Simulations using an anthropomorphic phantom}

Figure 1(a) shows the geometry used to simulate the full field irradiation of the breast. The anthropomorphic phantoms used were developed by Bakic et $\mathrm{al}^{6}$. Two breast phantoms were imaged at $0^{\circ}, 7.5^{\circ}$ and $25^{\circ}$ projection angles with compressed thicknesses of $5 \mathrm{~cm}$ and $7.5 \mathrm{~cm}$, and an average glandularity of $12 \%$ and $40 \%$ respectively. The composition of the adipose tissue, glandular tissue and skin within the phantom were taken from Hammerstein et $\mathrm{al}^{7}$, and the composition for Coopers ligaments was assumed to be that of adult skeletal muscle (ICRU Report $46(1992))^{8}$.

In contrast to much of the prior work, the edge of these phantoms mimic the curvature seen at the edge of real breasts, wherein an air gap exists between the lower skin surface of the breast phantom and the detector (See Figure 1(b)). This projected air gap and breast thickness along the ray's path have been calculated for each detector pixel using the methodology described by Siddon ${ }^{9}$. Each simulation used $10^{10}$ photons. The simulated X-ray spectrum was representative of a W/Rh target/filter combination at $29 \mathrm{kV}(\mathrm{HVL}=0.57 \mathrm{~mm} \mathrm{Al})$. The source-to-detector distance was $66 \mathrm{~cm}$ and the rotation centre for the DBT projections was placed $4 \mathrm{~cm}$ above an ideal detector.

\subsection{Scatter point spread response functions}

The scatter point spread response function (SPRF) was calculated for a series of uniform phantoms in a thickness range $(0.1$ to $10 \mathrm{~cm})$ and glandularities of $0 \%$ and $100 \%$ using a narrow pencil beam geometry as shown in Figure 1(c), which illustrates the position for three different projection angles. Also, the SPRF was recorded for an air gap $(A G)$ range from 0 to $5 \mathrm{~cm}$ in steps of $1 \mathrm{~mm}$.

As described by Sechopoulos et al ${ }^{10}$, the scatter point spread function (SPSF) becomes more asymmetric as the projection angle $\phi$ (perpendicular to the detector surface) increases. This phenomenon is illustrated in Figures 1(d) and 1(e). For this reason, the spatial distributions of the SPSFs were recorded using polar coordinates $(r, \theta)$ for different radii $r$ and angles $\theta$ in steps of $0.1 \mathrm{~mm}(\Delta r)$ and $3^{\circ}(\Delta \theta)$ respectively. Due to the low spatial frequency of the scatter, intermediate values have been calculated by linear interpolation. 


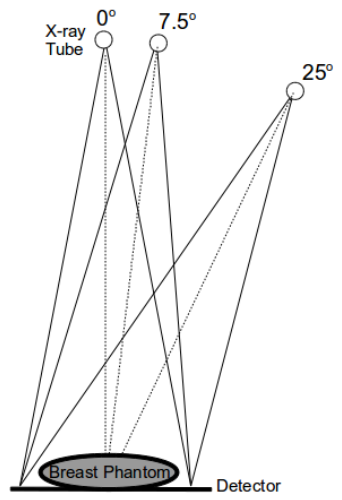

(a) DBT projections diagram

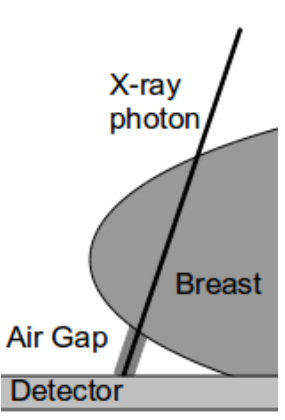

(b) Projected air gap

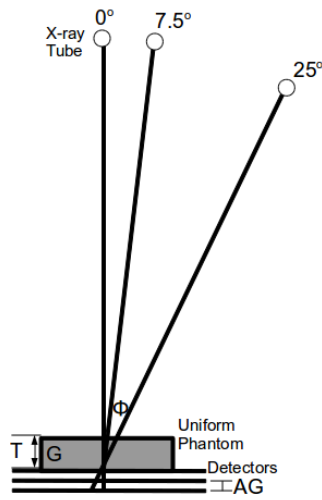

(c) Pencil beams diagram

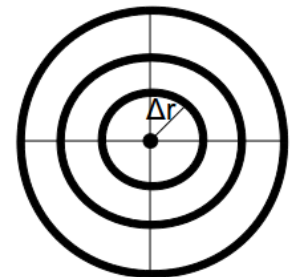

(d) $\operatorname{SPSF} \phi=0^{\circ}$

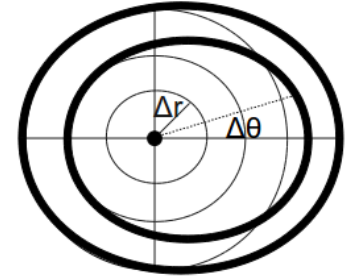

(e) $\operatorname{SPSF} \phi>0^{\circ}$

Figure 1. (a) Side-views of the DBT projections for $\phi=0^{\circ}, \phi=7.5^{\circ}$ and $\phi=25^{\circ}$. The air gap between the curved edge region of the breast and the detector is illustrated in (b). (c) Side-views of three pencil beam experiments for a uniform slab phantom of thickness $T$, glandularity $G$ and different air gaps $A G$. Top-view diagrams of scatter response for $\phi=0^{\circ}$ and $\phi>0^{\circ}$ are shown in (d) and (e) respectively. Note the isotropic scatter response for $\phi=0^{\circ}$ and the loss of circular symmetry for $\phi>0^{\circ}$ as the X-ray source tilts towards the left edge of the page.

\subsection{Scatter estimation}

It has been demonstrated that the SPSF varies mainly with breast thickness and projection angle accompanied by relatively little dependency on glandularity ${ }^{10-12}$. As described by several authors ${ }^{2,3}$, an estimate of the scatter distribution can be calculated by convolving the primary image with an appropriate scatter kernel allowing for thickness and projection angle. In these previous works, spatially invariant scatter kernels, which only account for breast thickness, have been considered. However, in the work presented here, the effect of the projected air gap traversed by photons exiting the curved edge of the breast phantom before impinging on the detector is also taken into account. For comparison of convolution based calculations with full MC calculations using the breast phantom, the primary image from full MC simulations was used. The scatter image $S(x, y)$ for a projection angle $\phi$, which now takes into account this air gap $A G$, is calculated as described below in Equation 1.

$$
\begin{gathered}
S(x, y)=\sum_{A G=0}^{A G_{\max }} \sum_{t=0}^{t_{\max }} P(x, y) \star \star K(z, z ; G ; t ; A G) \quad[k e V], \\
K_{G}(z, z ; t) \quad K_{G}(z, z ; t ; A G) \quad C \times K_{G}(z, z ; t ; A G)
\end{gathered}
$$

where $P(x, y)$ represents the primary image and $K(z, z ; G ; t ; A G)$ corresponds to the $2 \mathrm{D}$ kernel which is derived from the previously calculated $\operatorname{SPSF}(r, \theta)$. As mentioned previously, glandularity has a small effect on scatter, therefore $G$ represents the average glandularity of the breast phantom. Note that whilst the projected air gap is analytically calculable using a phantom of known geometry, the breast curvature would need to be estimated in the clinical situation. However, this is also true of any methodology which seeks to use such a kernel based approach.

\subsection{Relative SPR error map}

In order to investigate the results, the SPR images calculated from the MC primary and predicted scatter images $(\operatorname{SPR}(x, y))$ have been compared with SPR values calculated from direct MC simulations $\left(S P R_{M C}(x, y)\right)$, which are considered as ground truth data. A map of the SPR relative error for each pixel $\epsilon_{S P R}(x, y)$, has been created as described in Equation 3.

$$
\epsilon_{S P R}(x, y)=\frac{S P R_{M C}(x, y)-S P R(x, y)}{S P R_{M C}(x, y)} \times 100
$$




\subsection{Correction factor for scatter image}

For our purposes, an error of less than $10 \%$ for $\epsilon_{S P R}(x, y)$ is desirable. As will be seen in Section 3, although accounting for the physics processes at the breast edge produces an improvement over prior work, a further improvement is also required. A simple way to address this has been to introduce a residual correction factor, dependent on distance to the edge of the breast $(r)$. This has been calculated across the entire breast, and applied to the estimated scatter derived from Equation 1. This factor $(C(r))$ has been calculated pixel-by-pixel based for each projection as explained in Equation 4.

$$
C(r)=\frac{S_{M C}(x, y)}{S(x, y)}
$$

The scatter image after applying the correction factor $\left(S_{C}(x, y)\right)$ is calculated by multiplying $C(r)$ and the scatter calculated after the convolution stage $(S(x, y))$ as illustrated in Equation 5 .

$$
S_{C}(x, y)=S(x, y) \times C(r)
$$

\subsection{Scatter from the compression paddle}

In a further step, scatter from the compression paddle, which is a significant source of scatter ${ }^{10,13}$, has been calculated in order to include it as an additional component to the aforementioned scatter estimate. The scatter from the paddle is calculated using MC simulations and a similar geometry shown in Figure 1(a) but with a $2.4 \mathrm{~mm}$ polycarbonate slab on top of the breast, simulating the compression paddle. Then, ratios of scatter $\left(R_{S}(r)\right)$, across the entire breast projection, $\operatorname{with}\left(S_{W P}(x, y)\right)$ and without $\left(S_{N P}(x, y)\right)$ the compression paddle are observed and stored according to the minimum Euclidean distance from each pixel to the breast's edge $r$ (Figure 6).

$$
R_{S}(r)=\frac{S_{W P}(x, y)}{S_{N P}(x, y)}
$$

When adding the scatter from the paddle, each pixel of the estimated $\operatorname{scatter}(S(x, y))$ is multiplied with the appropriate ratio value $(R(r))$, according to its minimum Euclidean distance to the breast's edge.

In order to quantify the accuracy of this method, the relative error $\left(\epsilon_{P a d}(x, y)\right)$ between the scatter from a direct MC simulation with paddle $\left(S_{W P}(x, y)\right)$ and the scatter with no paddle after applying the aforementioned ratio is calculated, as observed in Equation 7.

$$
\epsilon_{P a d}(x, y)=\frac{S_{W P}(x, y)-\left(S(x, y) \times R_{S}(r)\right)}{S_{M C}(x, y)} \times 100
$$

\section{RESULTS}

Figure 2 shows the relative error in the SPR obtained when the conventional convolution-based approach described in ${ }^{1-3}$, then the proposed convolution, considering the air gap between the breast and detector, and finally the correction factor described in Section 2.5 was applied for imaging a $5 \mathrm{~cm}$ thick Bakic type phantom using a projection angle of $0^{\circ}$. For the conventional approach, only breast thickness and projection angles are considered, thus kernels with zero air gap are assumed. Profiles along the vertical white line are also shown in the figure. Large relative errors are observed near the edge of the phantom for the conventional approach (solid line). These errors are reduced when considering the air gap into account (blue crosses). However, as defined previously, errors less than $10 \%$ are desirable. In order to achieve this level of performance, a correction factor has been applied (Figure 3(a)), considerably reducing the error across the image (red circles). Note that only errors corresponding to regions inside the projected breast phantom are shown. Errors corresponding to regions outside the projected breast phantom are ignored. 


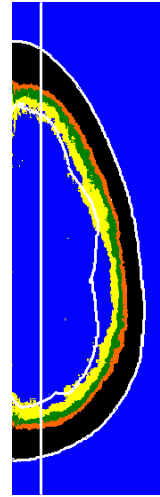

(a) Error map conventional convolut.

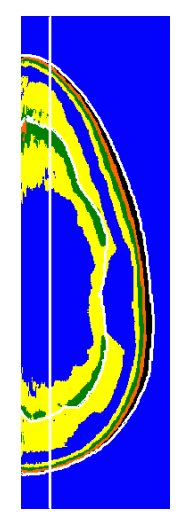

(b) Error map AG correction

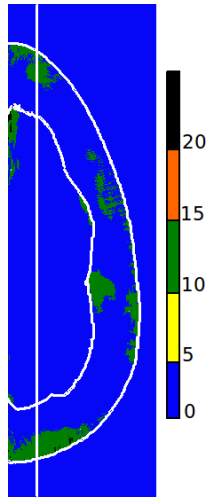

(c) Error map correction factor

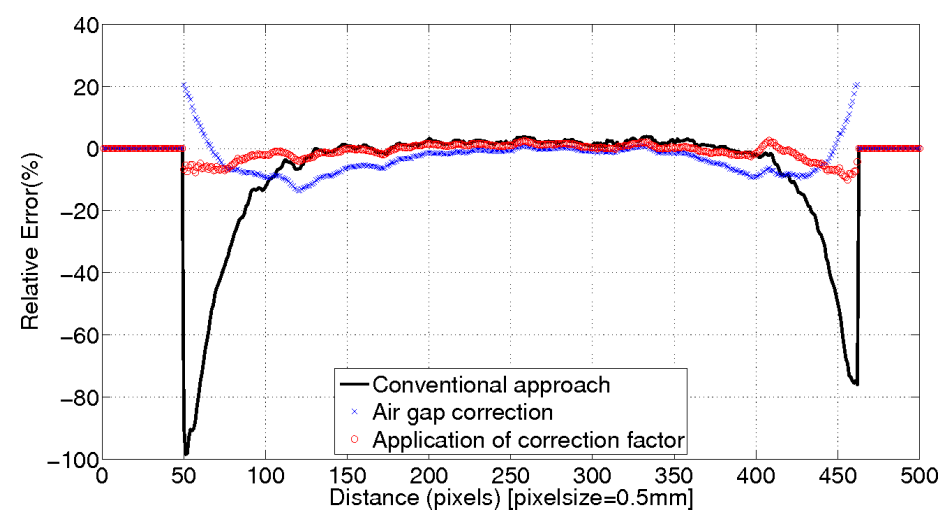

(d) Profiles of error maps

Figure 2. Relative error SPR maps $\left(\epsilon_{S P R}(x, y)\right)$ observed within a $5 \mathrm{~cm}$ breast phantom thickness and $\phi=0^{\circ}$ for estimated scatter using the conventional convolution-based approach (a), with the addition of the air gap correction (b) and also with the proposed correction factor applied (c). Note that errors are illustrated using a colormap, in \%, showing errors between 0 and greater than $20 \%$ in five discretised intervals. Profiles along vertical white lines are shown in (d). Edges where the air gap starts as well as the edges of the breast are highlighted as white contours.

These high discrepancies observed in the edges of the conventional approach are attributed to the the extended path length of scatter photons. At the skin edge, scatter photons project into air and no longer encounter the same scattering path length experienced whilst traversing solid scattering material, as illustrated in Figure 1(b).

As explained in Section 2.5, a simple correction factor (based on minimum distance to the breast edge) has been calculated in an attempt to decrease the errors observed. These correction factors, for $5 \mathrm{~cm}$ and $7 \mathrm{~cm}$ breast phantoms and the three projection studied in this document, are illustrated in Figure 3.

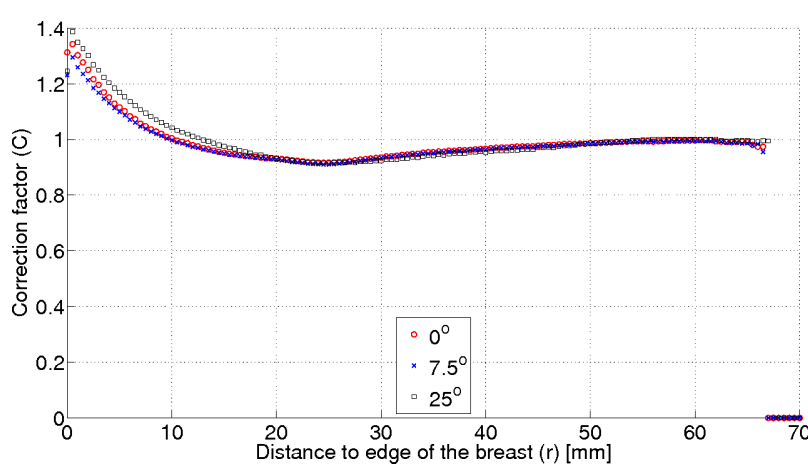

(a) Correction factor $5 \mathrm{~cm}$ breast

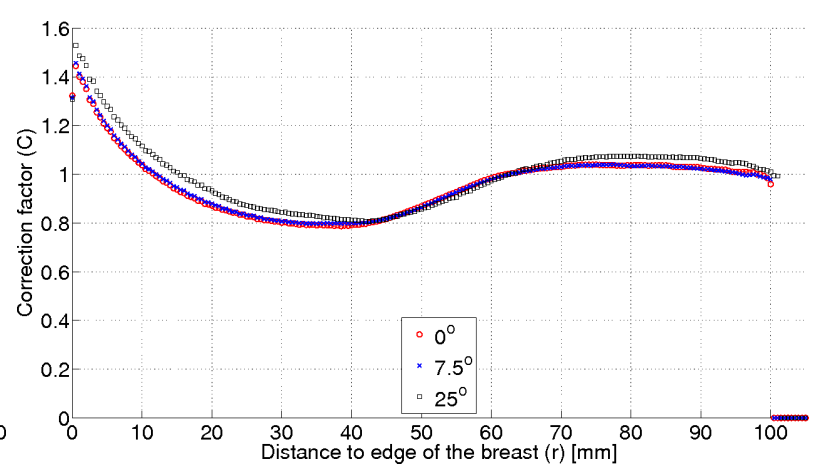

(b) Correction factor $7.5 \mathrm{~cm}$ breast

Figure 3. Correction factor values for breast thicknesses of $5 \mathrm{~cm}$ and $7 \mathrm{~cm}$ and incident angles of $0^{\circ}$ (circle), $7.5^{\circ}$ (cross) and $25^{\circ}$ (square).

Using the same $5 \mathrm{~cm}$ breast phantom, results of the scatter for the three methodologies explained above are shown in Figure 4 and 5 for exemplar projection angles of $7.5^{\circ}$ and $25^{\circ}$ respectively.

In these examples, larger errors are observed again near the edges of the phantom when using the conventional convolution, the extent and magnitude of which are dependent on incident beam angle and the projection of the asymmetric scatter field that this creates. Once more, the errors are reduced when using the proposed convolution which takes the air gap phenomena into account. However, the SPSFs are still unsatisfactory close to the edges, thus better results have been obtained when applying the aforementioned correction factor. 


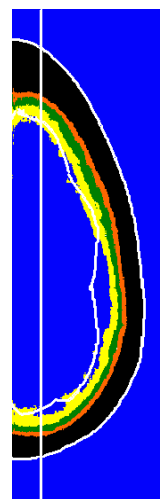

(a) Error map conventional convolut.

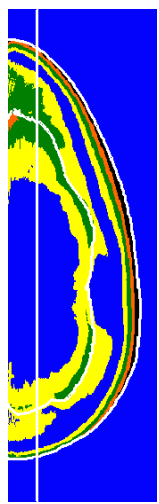

(b) Error map AG correction

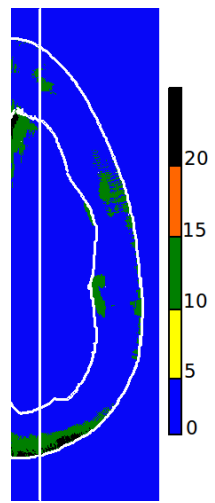

(c) Error map correction factor

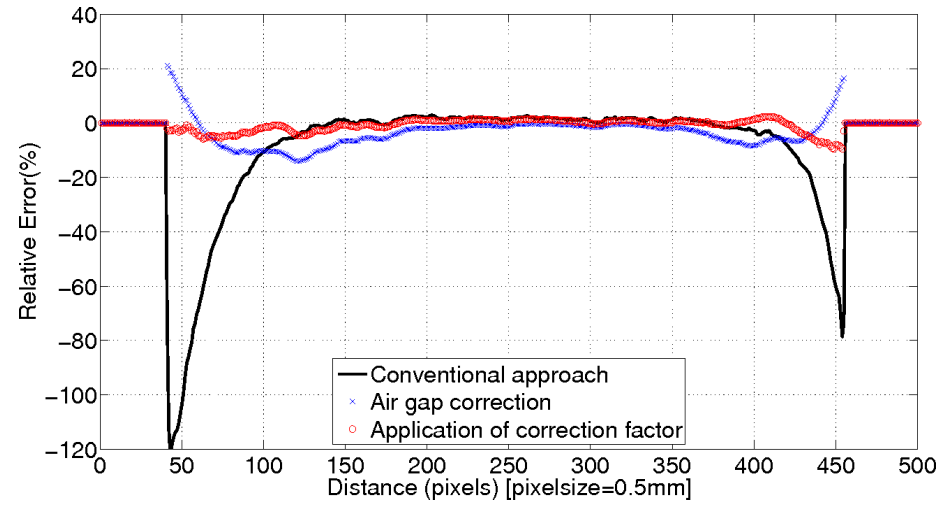

(d) Profiles of error maps

Figure 4. Relative error SPR maps $\left(\epsilon_{S P R}(x, y)\right)$ observed within a $5 \mathrm{~cm}$ breast phantom thickness and $\phi=7.5^{\circ}$ for estimated scatter using the conventional convolution-based approach (a), with the addition of the air gap correction (b) and also with the proposed correction factor applied (c). Note that errors are illustrated using a colormap, in \%, showing errors between 0 and greater than $20 \%$ in five discretised intervals. Profiles along vertical white lines are shown in (d). Edges where the air gap starts as well as the edges of the breast are highlighted as white contours.

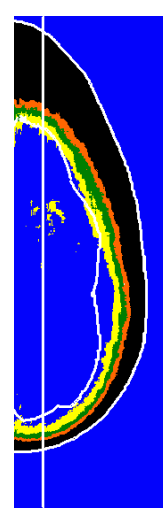

(a) Error map conventional convolut.

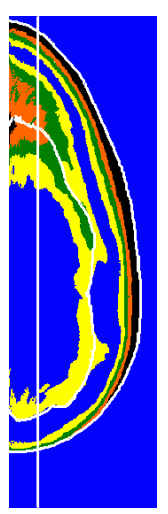

(b) Error map AG correction

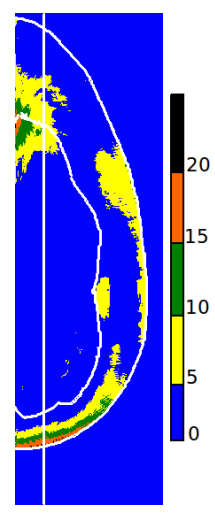

(c) Error map correction factor

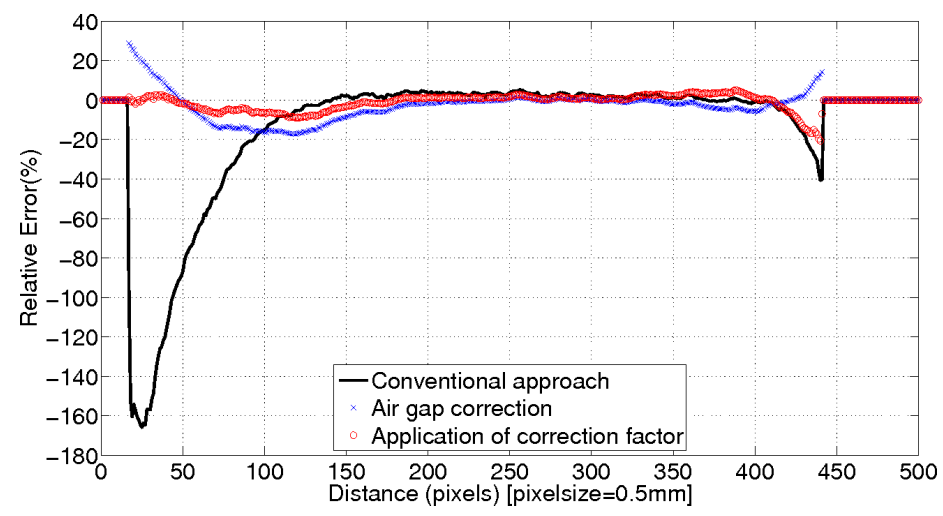

(d) Profiles of error maps

Figure 5. Relative error SPR maps $\left(\epsilon_{S P R}(x, y)\right)$ observed within a $5 \mathrm{~cm}$ breast phantom thickness and $\phi=25^{\circ}$ for estimated scatter using the conventional convolution-based approach (a), with the addition of the air gap correction (b) and also with the proposed correction factor applied (c). Note that errors are illustrated using a colormap, in \%, showing errors between 0 and greater than $20 \%$ in five discretised intervals. Profiles along vertical white lines are shown in (d). Edges where the air gap starts as well as the edges of the breast are highlighted as white contours.

Figures 6,7 and 8 illustrate the same analysis shown above but applied to a $7 \mathrm{~cm}$ breast phantom, as explained in the methodology section. For this phantom, we observed a similar trend as seen previously: errors are reduced when using the proposed convolution methodology to calculate the scatter but applying a correction factor produces a better result. However, it was found difficult to sustain errors lower than $10 \%$ in certain regions of the breast due to variations in local geometry. 


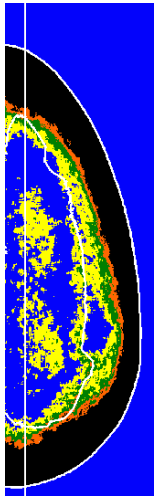

(a) Error map conventional convolut.

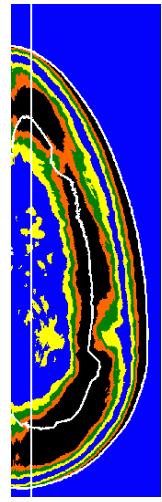

(b) Error map AG correction

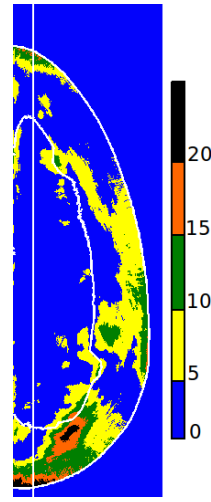

(c) Error map correction factor

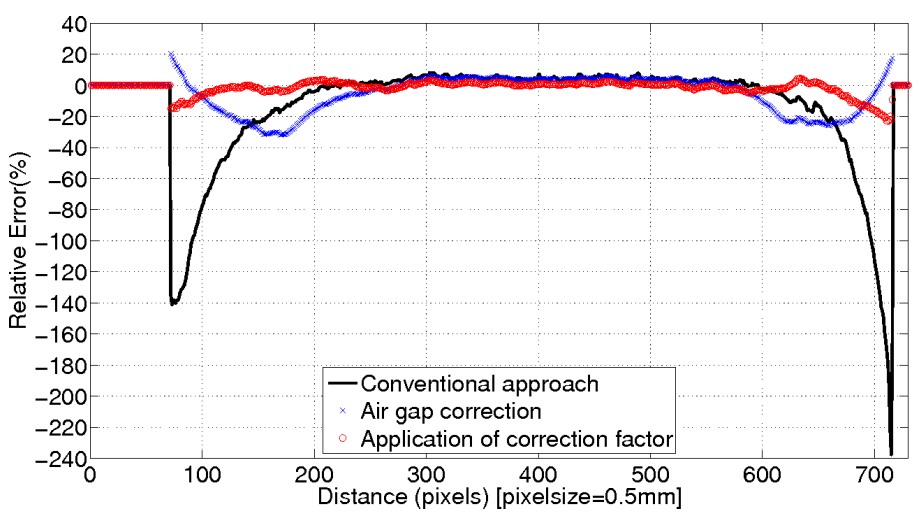

(d) Profiles of error maps

Figure 6. Relative error SPR maps $\left(\epsilon_{S P R}(x, y)\right)$ observed within a $7.5 \mathrm{~cm}$ breast phantom thickness and $\phi=0^{\circ}$ for estimated scatter using the conventional convolution-based approach (a), with the addition of the air gap correction (b) and also with the proposed correction factor applied (c). Note that errors are illustrated using a colormap, in \%, showing errors between 0 and greater than $20 \%$ in five discretised intervals. Profiles along vertical white lines are shown in (d). Edges where the air gap starts as well as the edges of the breast are highlighted as white contours.

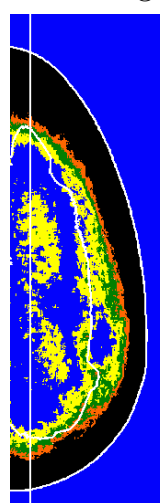

(a) Error map conventional convolut.

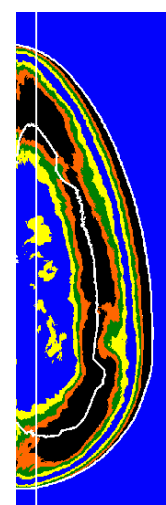

(b) Error map AG correction

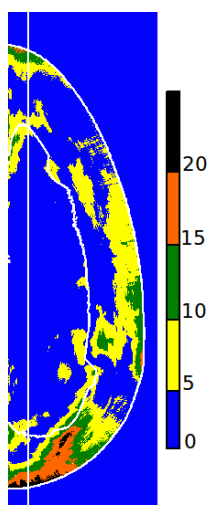

(c) Error map correction factor

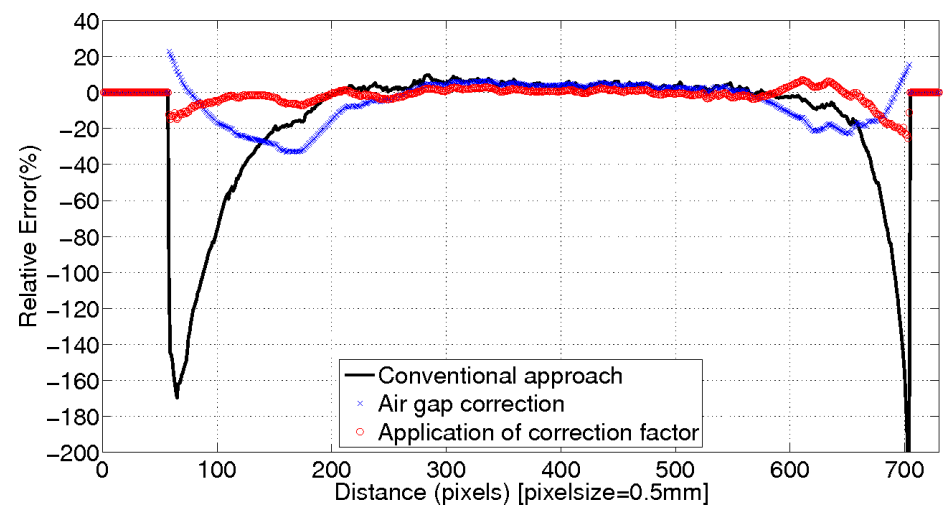

(d) Profiles of error maps

Figure 7. Relative error SPR maps $\left(\epsilon_{S P R}(x, y)\right)$ observed within a $7.5 \mathrm{~cm}$ breast phantom thickness and $\phi=7.5^{\circ}$ for estimated scatter using the conventional convolution-based approach (a), with the addition of the air gap correction (b) and also with the proposed correction factor applied (c). Note that errors are illustrated using a colormap, in \%, showing errors between 0 and greater than $20 \%$ in five discretised intervals. Profiles along vertical white lines are shown in (d). Edges where the air gap starts as well as the edges of the breast are highlighted as white contours.

Once the SPSFs are calculated, the convolution-based scatter estimation proposed here has reduced the time from approximately 12 hours (full MC simulations) to around a minute per projection. However, when the correction factor has been applied using a pixel-by-pixel approach, the execution time required rises to between 10 to 15 minutes, depending on the breast's size. However, some reduction in this figure may be anticipated via code optimisation. 


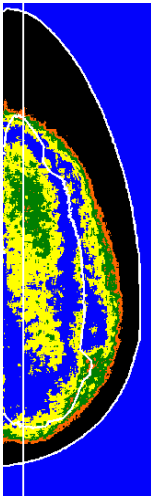

(a) Error map conventional convolut.

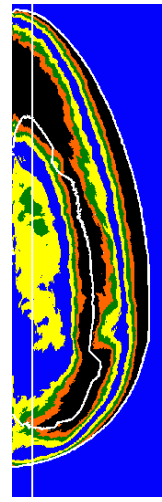

(b) Error map AG correction

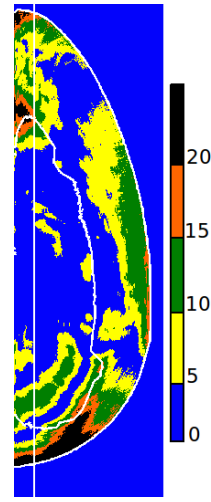

(c) Error map correction

factor

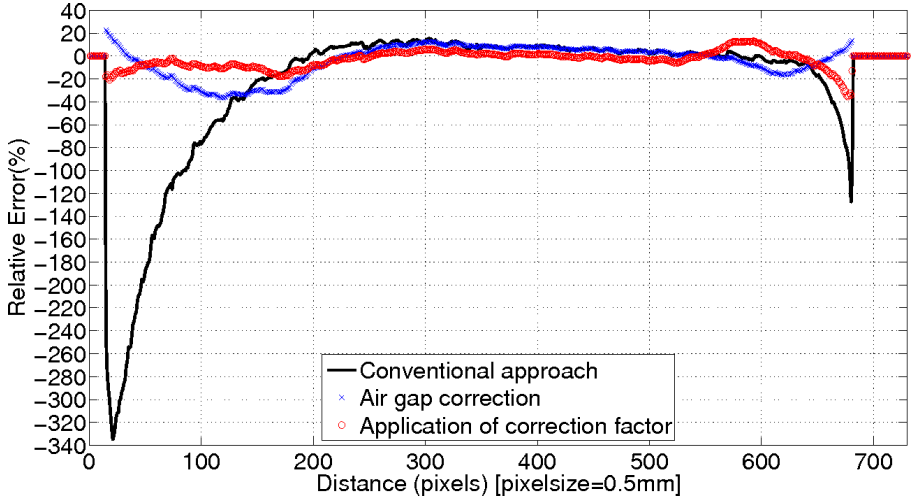

(d) Profiles of error maps

Figure 8. Relative error SPR maps $\left(\epsilon_{S P R}(x, y)\right)$ observed within a $7.5 \mathrm{~cm}$ breast phantom thickness and $\phi=25^{\circ}$ for estimated scatter using the conventional convolution-based approach (a), with the addition of the air gap correction (b) and also with the proposed correction factor applied (c). Note that errors are illustrated using a colormap, in \%, showing errors between 0 and greater than $20 \%$ in five discretised intervals. Profiles along vertical white lines are shown in (d). Edges where the air gap starts as well as the edges of the breast are highlighted as white contours.

As described in the methodology section, scatter from the compression paddle has been also studied in this research. The scatter ratio, $R_{S}$, is illustrated in Figure 9(a). After the ratio is applied to the estimated scatter radiation without paddle (to insert the scatter from the paddle), the relative error $\epsilon_{P a d}(x, y)$ across the whole image, as well as a profile (solid red line) is shown in Figures 9(b) and 9(c) respectively. Moreover, for comparison, the same profiles is shown for an image without paddle correction. As expected, large errors observed in the no paddle images have been reduced when applying the aforementioned $R_{S}$.

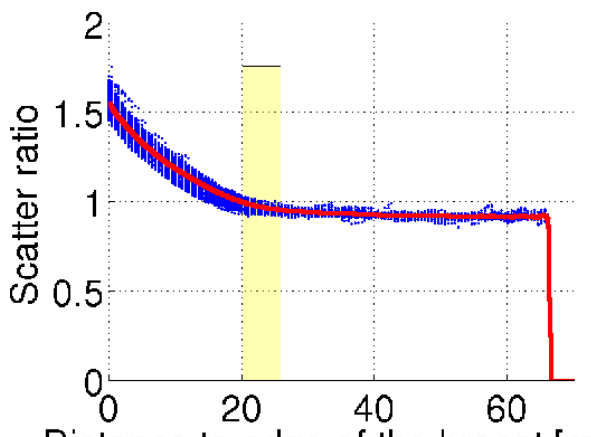

Distance to edge of the breast [mm]

(a) Ratio

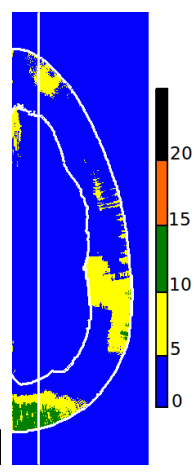

(b) Error

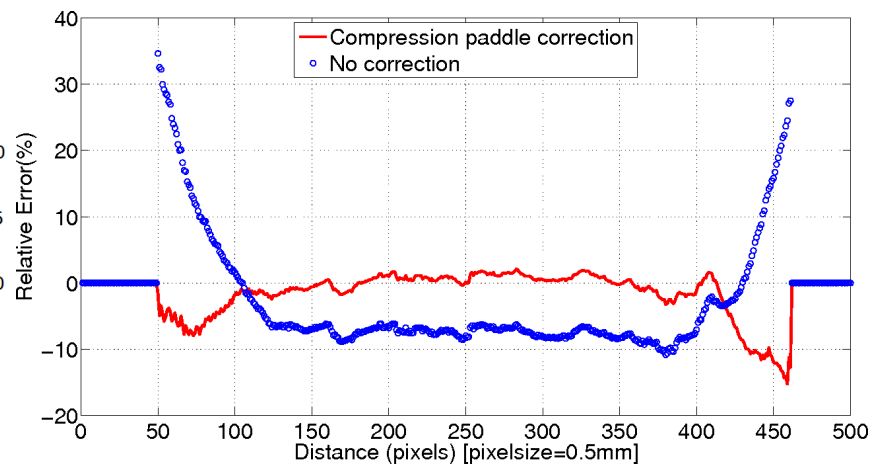

(c) Profile

Figure 9. Scatter from compression paddle: (a) represents the ratio $R_{S}(r)$ for a $5 \mathrm{~cm}$ breast phantom and $0^{\circ}$ incidence angle. Each single point of the ratio between the scatter with and without compression paddle is shown as overlying blue dots while the average value is plotted as a red solid line. The variation in the distance from the constant thickness region to the edge of the breast is shown as a yellow bar, centred at the average distance with a width representing two standard deviations. The relative error $\epsilon_{P a d}(x, y)$ is illustrated in (b) whereas a vertical profile is presented as a solid red line in (c). The blue circles in the profile correspond to the predicted scatter image before applying the scatter from paddle correction. 


\section{DISCUSSION AND CONCLUSIONS}

A detailed study of fast scatter calculation for DBT using a voxelised breast phantom is presented for the first time. The use of the voxelised breast phantom provides more realistic results when studying physical processes such as scatter radiation.

Good agreement between calculated and MC SPR at the central region of the breast phantom shows that a conventional scatter kernel successfully accounts for scatter in the regions of constant breast thickness. Although the decreasing thickness of the breast is accounted for in the kernel, the method still generates significant errors (over 50\%) in the peripheral non-constant thickness region, where there is an air gap below the curved breast edge. Furthermore, it was observed that such errors increase with projection angle as the geometry exacerbates the scatter asymmetry in this region.

Here, a new method is proposed to correct for such non-uniform regions of the breast by using a kernel which takes into account the air gap between the phantom and detector. Accounting for the air gap in the non-uniform thickness region produced a significant reduction in the error of the estimated scatter by approximately a factor of 5 . However, accounting for the scattering phenomena in this region, whilst producing a dramatic reduction in error, did not achieve the target error of less than $10 \%$. This is due to the fact that where the thickness is not constant, SPSF kernels from large area phantoms have been used to maintain execution speed.

In order to improve the above methodologies, a simple correction factor is proposed. Results showed this final correction stage produced a reduction in scatter for the $5 \mathrm{~cm}$ breast phantom to within $10 \%$ error. However, certain regions with relative errors larger than $10 \%$ were found for the case of $7 \mathrm{~cm}$ breast phantom.

Finally, an attempt to insert scatter from the compression paddle in an image calculated without the compression paddle is shown. Overall, good agreement was found across the breast when comparing the estimated results with the ground truth data from MC simulations. As a next step, this will be extended to other projection angles and breast thicknesses.

\section{ACKNOWLEDGMENTS}

This work is part of the OPTIMAM project and is supported by CR-UK \& EPSRC Cancer Imaging Programme in Surrey, in association with the MRC and Department of Health (England).

\section{REFERENCES}

[1] Wu, G., Mainprize, J., Boone, J., and Yafee, M., "Evaluation of scatter effects on image quality for breast tomosynthesis," Medical Physics 36(10), 4425-4432 (2009).

[2] Boone, J. and Cooper, V., "Scatter/primary in mammography: Monte Carlo validation," Medical Physics 27(8), 1818-1831 (2000).

[3] Ducote, J. and Molloi, S., "Scatter correction in digital mammography based on image deconvolution," Physics in Medicine and Biology 55, 1295-1309 (2010).

[4] Agostinelli, S., Allison, J., Amako, K., Apostolakis, J., Araujo, H., Arce, P., Asai, M., Axen, D., Banerjee, S., and Barrand, G., "Geant4 - a simulation toolkit," Nuclear Instruments and Methods in Physics Research 506(3), 250-303 (2003).

[5] Allison, J., Amako, K., Apostolakis, J., Araujo, H., Arce, P., Asai, M., Barrand, G., Capra, R., and Chauvie, S., "Geant4 development and applications," IEEE Transactions on Nuclear Science 53(1), 270-278 (2006).

[6] Bakic, P., Zhang, C., and Maidment, A., "Development and characterization of an antropomorphic breast software phantom based upon region-growing algorithm," Medical Physics 38(6), 3165-3176 (2011).

[7] Hammerstein, G., Miller, D., White, D., Masterson, M., Woodard, H., and Laughlin, J., "Absorbed radiation dose in mammography," Radiology 130, 485-491 (1979).

[8] ICRU, "Photon, electron, proton and neutron interaction data for body tissues," Tech. Rep. 46, International Commission on Radiation Units and Measurements (1992). 
[9] Siddon, R., "Fast calculation of the exact radiological path for a three-dimensional CT array," Medical Physics 12(2), 252-255 (1985).

[10] Sechopoulos, I., Suryanarayanan, S., Vedantham, S., D'Orsi, C., and Karellas, A., "Scatter radiation in digital tomosynthesis of the breast," Medical Physics 34(2), 564-576 (2007).

[11] Boone, J., Lindfors, K., Cooper, V., and Seibert, J., "Scatter/primary in mammography: Comprehensive results," Medical Physics 27(10), 2408-2416 (2000).

[12] Cooper, V., Boone, J., Seibert, J., and Pellot-Barakat, C., "An edge spread technique for measurement of the scatter-to-primary ratio in mammorgaphy," Medical Physics 27(5), 845-853 (2000).

[13] Dance, D. and Day, G., "The computation of scatter in mammography by Monte Carlo methods," Physics in Medicine and Biology 29(3), 237-247 (1984). 